\title{
The Impact of Ultrasound on APP/PS1 Model Mouse
}

\author{
$\mathrm{Li} \mathrm{Li}^{\mathrm{a}}$, Weiwei Wang ${ }^{\mathrm{b}}$, Wei Wu ${ }^{\mathrm{c},}{ }^{\star}$, Wanlin Zhang ${ }^{\mathrm{d}}$, Ying Gao ${ }^{\mathrm{e}}$ \\ and Chen Chen ${ }^{f}$ \\ School of Southeast University, Jiangsu 210009, China \\ a1404157708@qq.com, b1715556404@qq.com, ${ }^{c} 101005800 @$ seu.com, d1292665976@qq.com, \\ e3236027533@qq.com, ${ }^{9} 915666024 @ q q . c o m$
}

Keywords: Alzheimer's disease, Amyloid plaques, Ultrasound.

\begin{abstract}
In this study, three-month-old APP / PS1 double transgenic mice were subjected to ultrasound intervention for investigating the effect of ultrasound intervention. The Morris water maze test showed that the ability of learning and memory of US group was significantly improved than that of the $\mathrm{AD}$ group $(\mathrm{p}<0.05)$, during the navigation and space exploration phases. The amyloid plaque content in the brain was detected by immunohistochemistry. The deposition of amyloid plaques in the brain of the US group was less than that in the AD group, but there was no significant difference between the $\mathrm{CN}$ group and the US group. The results showed that the learning and memory ability of APP / PS1 double transgenic mice was significantly improved after the intervention of ultrasound with frequency of $1 \mathrm{MHz}$ and power of $0.3 \mathrm{~W} / \mathrm{cm}^{2}$, and the amyloid plaques were reduced.
\end{abstract}

\section{Introduction}

Alzheimer's disease [1] is an age-associated neurodegenerative disorder, owing to the death of central nervous cells. Primary manifestations of Alzheimer's disease include cognitive impairment, alterations in behavior, daily life ability disorder, eventually bedridden and incontinence.

Nonpharmacologic and pharmacologic interventions are available, although none prevents or cures the disease. Pharmacologic therapies are intended to slow the progression of disease and improve symptoms. Drugs [2] currently approved for Alzheimer's include cholinesterase inhibitors (donepezil hydrochloride [donepezil], galantamine hydrochloride [galantamine], rivastigmine tartrate [rivastigmine], and tacrine hydrochloride [tacrine] and memantine, an N-methyl-D-aspartate (NMDA) receptor antagonist. Although these drugs have been approved for listing, researchers did not stop verifying the efficacy of approved drugs and further exploring AD treatment method.

The advantages of ultrasound stimulation are that it does not necessitate surgery or genetic alteration, but it confers spatial resolutions superior to other noninvasive methods such as transcranial magnetic stimulation [3].

Ultrasound in the medical field is developing rapidly, for example, induced focused ultrasound damage, clear protein aggregates, open the blood-brain barrier then regulate neuronal function and other increasingly diverse new applications, expanding the application of medical ultrasound in the field of nervous system $[4,5]$.

Activated glial cells increase the clearance of amyloid by swallowing amyloid, transcranial ultrasonography can activate microglia, thereby promoting its ingestion of amyloid [6]. Studies have shown that microglia and astrocytes are activated in the cortical region after irradiation with focused ultrasound $(\mathrm{f}=1 \mathrm{~Hz})$.

Therefore, we hypothesized that ultrasound intervention can improve the APP/PS1 double transgenic mice's learning and memory ability and reduce the amyloid plaques. The main objective of this study evaluate the effectiveness of ultrasound intervention on APP / PS1 double transgenic mice, which provides a reference for application prospects of the Ultrasound technology for AD intervention. 


\section{Materials and methods}

\subsection{Animals.}

The research shows that APP / PS1 double transgenic mice[7,8] A $\beta 42$ levels were elevated at the third month of age, 5 months or even earlier appeared deposition of senile plaques in the brain, in 9 months with a large number of senile plaques in the brain. The presence of amyloid plaques in the cerebral cortex and hippocampus of mice increased with age. This is consistent with the development and symptoms of human AD.

Twenty APP / PS1 double transgenic mice and ten non-transgenic control mice (Beijing Huafu Kang Biological Technology Co., Ltd, SCXK (Beijing) 2014-0004, No.11401300029102) were used in this study, Male, three months old, weighing 20-22g.

Among these twenty APP / PS1 double transgenic mice were divided into the following two experimental groups with $n=10$ for each group. (1) AD group: no Ultrasonic treatment. (2)US group: with ultrasonic treatment. The remaining ten non-transgenic control mice were group 3 following the same treatment as the control group.

\subsection{Ultrasonic radiation parameters and methods.}

According to the characteristics of the experiment, the safety and the reference literature, the parameters of the ultrasonic intervention of APP / PS 1 double transgenic mice were $\mathrm{P}=0.3 \mathrm{~W} / \mathrm{cm}^{2}$, $\mathrm{f}=1 \mathrm{~Hz}$, daily treatment for $10 \mathrm{~min}$, sustained $6 \mathrm{w}$, remove the mouse head hair with a depilatory cream in the first intervention. Before each intervention, the medical ultrasonic coupling agent was applied to the mice head to ensure that the ultrasound probe was closely coupled with the mice head.
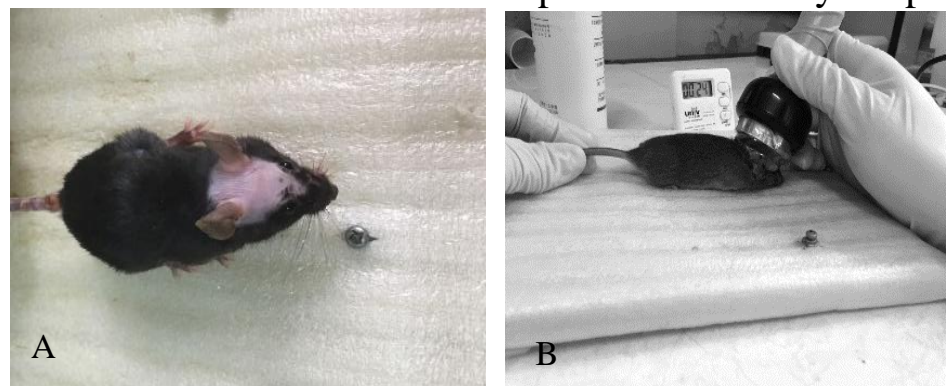

\section{$\begin{array}{lll}\text { A. After hair removal } & \text { B. Ultrasound treatment of mice }\end{array}$}

Figure 1. Ultrasonic intervention

\subsection{Evaluation method.}

\subsubsection{Morris water maze test $[9,10]$.}

The Morris water maze (MWM) is a test of spatial learning for rodents that relies on distal cues to navigate from start locations around the perimeter of an open swimming arena to locate a submerged escape platform. Spatial learning is assessed across repeated trials and reference memory is determined by preference for the platform area when the platform is absent. Evaluation of the indicators are: track; swimming speed; from the release to the mouse to climb the underwater platform swimming time.

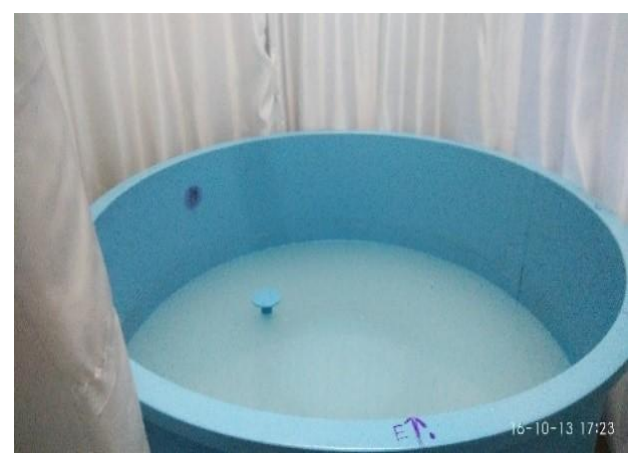

Figure 2. Water maze

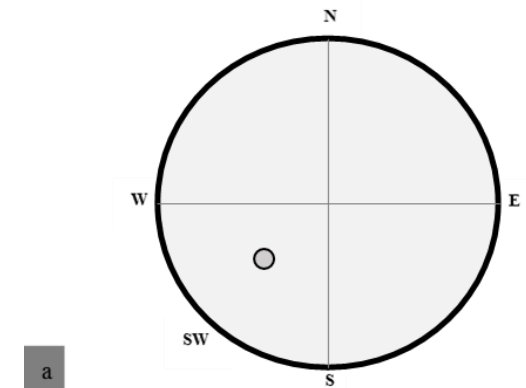

Figure 3. Water maze quadrant and platform distribution 


\subsubsection{Immunohistochemistry.}

Rats were sacrificed by deep anesthesia using 3\% pentobarbital and perfused through the heart with cold phosphate-buffered saline (PBS), followed by $4 \%$ paraformaldehyde. Brains were removed from the skull on the ice, and post-fixed in the $4 \%$ paraformaldehyde for $48 \mathrm{~h}$. And then followed by water rinse with water, alcohol dehydration transparent, dip wax, embedding, slicing, mounting and baking sheets, the slices at $65^{\circ} \mathrm{C}$ dry $(1 \mathrm{~h})$.

\subsection{Statistical analysis.}

The experimental data were organized with office excel 2013, and then computer-analyzed using SPSS program. Data are expressed as mean \pm SEM. Statistical analysis was conducted by one-way analysis of variance (ANOVA), followed by Bonferroni or SNK. A P-value of less than 0.05 was considered statistically significant.

\section{Results}

\subsection{Morris water maze test}

The Morris water maze (MWM) is a test of spatial learning and Short-term memory for rodents.

After six weeks of treatment, the three groups of mice were subjected to a six-day Morris water maze test. According to the executive rule, since the first day of the mouse was in the early stage of the semester, most mice did not find the hidden platform in time, and the results of the first day were not statistically analyzed. The statistical analysis of the relevant indicators that the first 2-5 days of navigation stage and the 6th day of the space exploratory stage was carried out.

The first section was the positioning of navigation experiments, detection of mouse space learning ability. Statistical analysis was conducted by one-way analysis of variance (ANOVA). With the increase in the number of training days, the three groups of mice in the specified time find the correct rate of hidden platform increased, the latency of the search platform is decreasing. On the third day, the latency of the $\mathrm{CN}$ group was shorter than that of the AD group $(\mathrm{p}<0.05)$, on the fourth day, the latency of the US group was shorter than that of the AD group $(p<0.05)$, there was no significant difference in the latency that seek the hidden platform between the $\mathrm{CN}$ group and the US group ( $\mathrm{p}>$ 0.05), as shown in Figure 4. As shown in Figure 5, there was no statistically significant difference in swimming between the three groups $(\mathrm{F}=1.156, \mathrm{p}>0.05)$. From the above, indicating that the overall reducing trend of the latency and the time difference between groups is not caused by swimming speed.

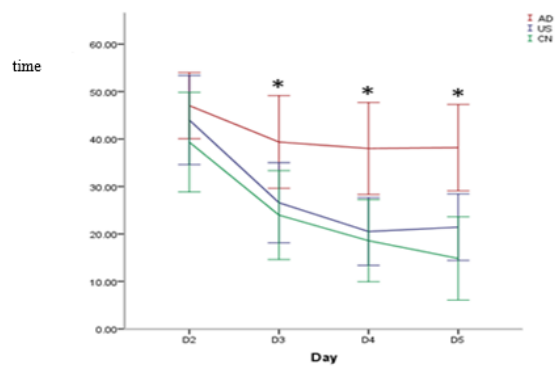

Figure 4. The latency looking for

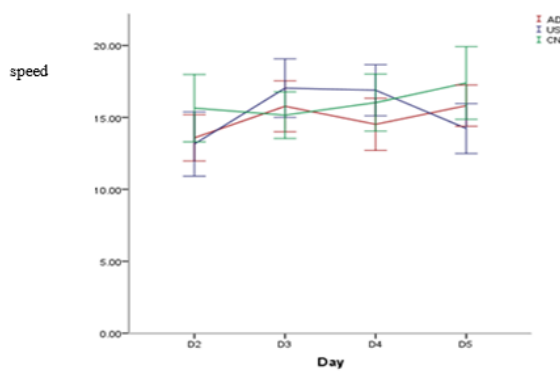

Figure 5. The swimming speed hidden platform latency

From the action track, the search strategy of the hiding platform of the mouse is from the edge of the "aimless", the random to the trend, the linear transformation, and with the training time, this trend is more obvious, as shown in Figure 6. 


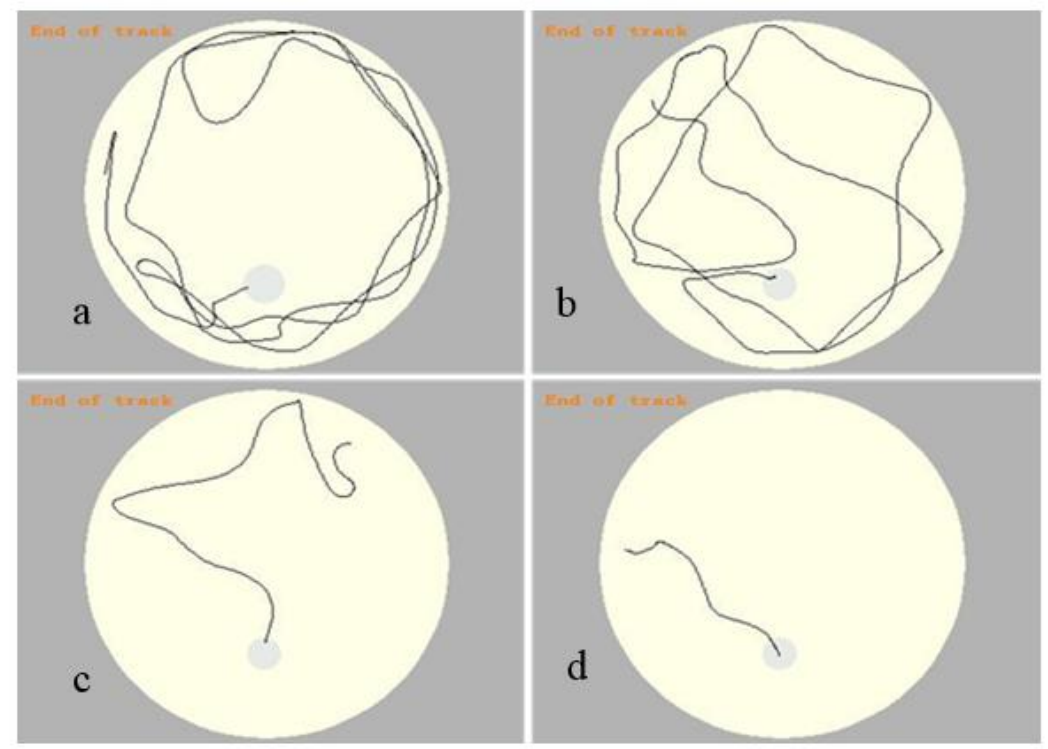

Figure 6. The diagram of searching strategy typical trajectory

The second stage, space exploration stage detect mouse spatial memory. After the hidden platform was removed, its residence time at the target quadrant were recorded and analyzed to detect the memory ability of the mouse. Statistical analysis was conducted by one-way analysis of variance (ANOVA) showed that there was a significant difference in the residence time of SE quadrant in the three groups $(\mathrm{F}=6.905, \mathrm{p}<0.05)$. As shown in Figure 7 , AD group compared with $\mathrm{CN}$ group in SE quadrant residence time was significantly reduced $(\mathrm{p}<0.05)$, AD group than the US group in the SE quadrant of the residence time is also significantly reduced $(\mathrm{p}<0.05)$, there was no significant difference in the residence time of SE quadrant between the US group and the CN group ( $p>0.05)$. As shown in Figure 8, there was no difference in swimming speed between the three groups $(\mathrm{F}=1.439$, $\mathrm{p}>0.05)$.

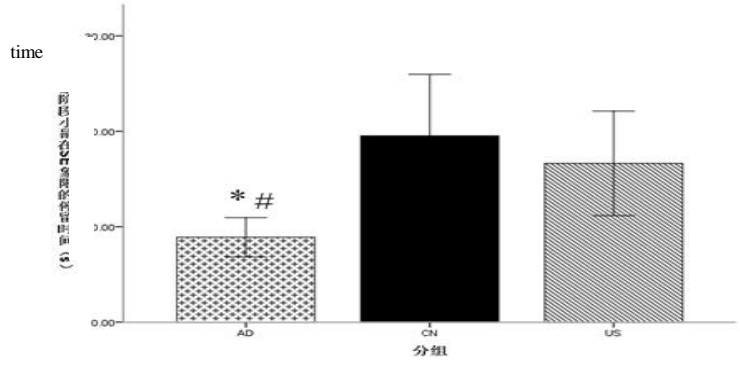

Figure 7. The time of stay in the SE quadrant

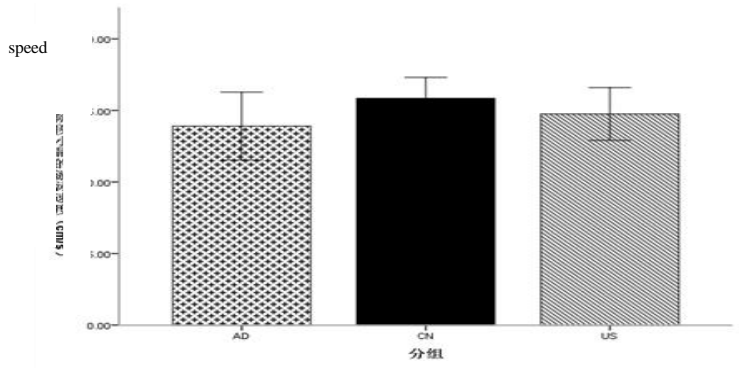

Figure 8. The swimming speed

There was no difference in swimming speed between the groups of mice during the navigation and space exploration phases. In addition, ultrasound intervention did not affect the athletic ability and visual acuity of the mice. The water maze can be more objective and scientific evaluation of the ability of learning and memory of mice. The experiments of locating navigation and space exploration show that the ultrasound intervention can improve the spatial learning and memory ability of APP / PS1 double transgenic mice.

\subsection{Immunohistochemistry.}

The mice's behavior test was performed after the intervention, and then the amyloid plaque content in the brain was detected by immunohistochemistry. The results showed that amyloid deposition was not found in the hippocampus of the CN group and the US group, and a small amount of amyloid deposition occurred in the brain of the AD mice in the 4.5-month-old AD group, this is consistent with the presence of sedimentary amyloid plaques in the brain of APP / PS1 mice around the age of four months found in the relevant study [11-12], as shown in Figure 9. 

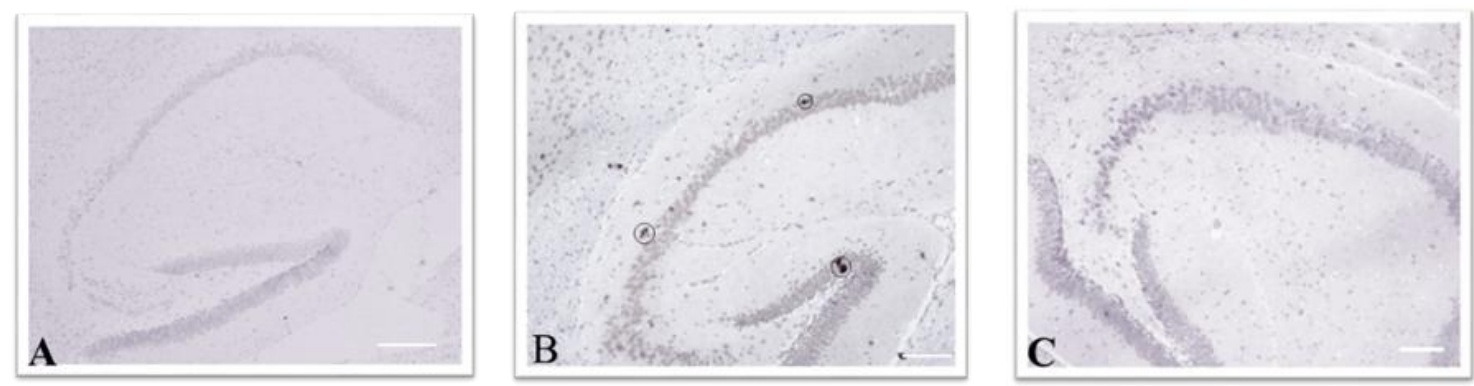

Figure 9. A The region of $\mathrm{CN}$ group mice hippocampus; B The region of AD group mice hippocampus; C: The region of US group mice hippocampus

\section{Discussion}

At this stage the main medical point of view for the amyloid plaque hypothesis[13,14]: an imbalance between production and clearance of Ab42 was initiating factor in Alzheimer's disease (AD), persistent deposition of amyloid plaques further leads to Tau protein fibron tangles, neuronal degeneration, and dementia. Genetics, physiology, biochemistry and behavioral studies have shown that $\mathrm{A} \beta 42$ plays a main role in the pathogenesis of $\mathrm{AD}[14,15]$. Aged plaques are formed by extracellular deposition of A $\beta 42$-like proteins, these aggregates impair neuronal activity by severely disrupting nerve fibers, causing serious damage to cell bodies and axons [16]. In conclusion, the ultimate occurrence of $\mathrm{AD}$ is closely related to the long-term imbalance between $\mathrm{A} \beta 42$ production and clearance.

Transcranial ultrasound is a safe and effective technique for regulating brain function, for example, low-frequency focused ultrasound can act on specific regions of the brain to alter the electrophysiology and synaptic plasticity of experimental animals [3]. Ultrasound can also regulate neuronal activity, and even promote the brain's advanced cognitive function and awareness of the improvement. In the hippocampus region of mice, low-frequency ultrasound can regulate neuronal activity by influencing sodium and calcium channels [17]. In the motor area of the cerebral cortex, ultrasound can stimulate neurons and trigger the activity of mice. Ultrasound stimulation of the subcortical deep region, the same can cause changes in neuronal activity and hippocampal structural vibration, at the same time, and did not observe the increase in the target area temperature anomalies [18]. Angelika Alonso et al, showed that cell albumin was phagocytosed after $30 \mathrm{~min}$ of ultrasound treatment, and the effect was still effective after $24 \mathrm{~h}$, the activated microglia played a major role; at the same time, the study found that activation of microglia at the same time, did not cause neuronal death and glial cell damage, which Streit et al's findings are consistent, that ultrasound can be a safe way to activate the nerve glue Cytokines $[19,20]$.

The central nervous system immune response contributes to the reduction of amyloid protein in the brain [21]. Clinical imaging and neuropathological studies have shown that microglia in the central nervous system can maintain the normal physiological activity of the brain, and the activated microglia can protect the central nervous system [22]. Microglia is closely related to amyloid in AD patients and in the brain of transgenic mice that produce amyloid plaques [23,24] There are many microglia around their amyloid plaques [25]. Simard et al ,observed that microglial cells in the activated state invade into the deposited amyloid plaques, which were also found in other experiments, and this phenomenon of invasion occurred after the formation of amyloid plaques, that is , sedimentary amyloid plaques induce microglia invasion, At the same time, experiments with APP / PS1 mice showed that microglia in the activated state could swallow amyloid plaques deposited in the central nervous system [26],transcranial ultrasonography can activate microglia, thereby promoting its ingestion of amyloid. Studies have shown that microglia and astrocytes are activated in the cortical region after irradiation with focused ultrasound $(f=1 \mathrm{~Hz})$, and their activation is manifested as an increase in the size and shape of the glial cells, whereas the number of cells is not increase. Activated glial cells increase the clearance of amyloid by swallowing amyloid. In addition, there is another 
similar study that the use of ultrasound treatment $(\mathrm{f}=12 \mathrm{MHz}, \mathrm{t}=3 \mathrm{~min})$ in vitro the microglia cells, morphological observation of increased in the size and shape of the glial cells, indicating that microglia can be activated by ultrasound [6], activated glial cells may be swallowing amyloid, thereby reducing amyloid plaques.

Animal experiment model in line with the development of human disease, the effectiveness of the study can be used as an important part of the medical device animal test, that is, the actual use of medical equipment and intended use to meet the compliance.

The subjective of this study was APP / PS1 double transgenic mice, an animal model that conformed to the development of the typical pathologic features of human AD. The animal's experimental results support further clinical trials. And the entire experimental process and experimental conditions are also in line with the norms, therefore, the study can simultaneously evaluate the effectiveness of interventions. Which provides a reference for the application of ultrasound in the intervention of $\mathrm{AD}$.

\section{Summary}

The results showed that the learning and memory ability of APP / PS1 double transgenic mice was improved after the intervention of ultrasound with frequency of $1 \mathrm{MHz}$ and power of $0.3 \mathrm{~W} / \mathrm{cm}^{2}$, and the amyloid plaques were reduced.

\section{References}

[1]. Tan C C, Yu J T, Wang H F, et al. Efficacy and safety of donepezil, galantamine, rivastigmine, and memantine for the treatment of Alzheimer's disease: a systematic review and meta-analysis. J Alzheimers Dis. 2014. 41(2): 615-631.

[2]. Tufail Y, Yoshihiro A, Pati S, et al. vb. Nature Protocols. 2011. 6(9): 1453-1470.

[3]. Scheltens P, Blennow K, Breteler M M B, et al. Alzheimer's disease. Lancet. 2016.388(10043):505-517.

[4]. Leinenga $G$, Langton $C$, Nisbet R, et al. Ultrasound treatment of neurological diseases -current and emerging applications. Nature Reviews Neurology. 2016. 12(3): 161-174.

[5]. Simard A R, Soulet D, Gowing G, et al. Bone Marrow-Derived Microglia Play a Critical Role in Restricting Senile Plaque Formation in Alzheimer's Disease. Neuron. 2006. 49(4): 489-502.

[6]. Janota C S, Brites D, Lemere C A, et al. Glio-vascular changes during ageing in wild-type and Alzheimer's disease-like APP/PS1 mice. Brain Research. 2015. 1620: 153-168.

[7]. Filali M, Lalonde R, Theriault $\mathrm{P}$, et al. Cognitive and non-cognitive behaviors in the triple transgenic mouse model of Alzheimer's disease expressing mutated APP, PS1, and Mapt, (3xTg-AD)[J]. Behavioural Brain Research, 2012, 234(2):334-342.

[8]. Vorhees C V, Williams M T. Morris water maze: procedures for assessing spatial and related forms of learning and memory. Nature Protocols. 2006. 1(2): 848-858.

[9]. D Hooge R, De Deyn P P. Applications of the Morris water maze in the study of learning and memory. Brain Research Reviews. 2001. 36(1): 60-90.

[10]. Pedrós I, Petrov D, Allgaier M, et al. Early alterations in energy metabolism in the hippocampus of APPswe/PS1dE9 mouse model of Alzheimer's disease. Biochimica et Biophysica Acta (BBA) - Molecular Basis of Disease, 2014, 1842(9): 1556-1566.

[11]. Dellu F, Contarino A, Simon H, et al. Genetic Differences in Response to Novelty and Spatial Memory Using a Two-Trial Recognition Task in Mice. Neurobiology of Learning and Memory, 2000, 73(1): 31-48.

[12]. Selkoe D J, Hardy J. The amyloid hypothesis of Alzheimer's disease at 25 years. EMBO Mol. Med. 2016. 8(6): 595-608.

[13]. Mullard A. Alzheimer amyloid hypothesis lives on. Nat Rev Drug Discov. 2016. 16(1): 3-5.

[14]. O’ Brien R J, Wong P C. Amyloid Precursor Protein Processing and Alzheimer's Disease. Annual Review of Neuroscience. 2011. 34(1): 185-204. 
[15]. Sepúlveda F J, Fierro H, Fernandez E, et al. Nature of the neurotoxic membrane actions of amyloid- $\beta$ on hippocampal neurons in Alzheimer's disease. Neurobiology of Aging. 2014. 35(3): 472-481.

[16]. Tyler W J, Tufail Y, Finsterwald M, et al. Remote excitation of neuronal circuits using low-intensity, low-frequency ultrasound. PLoS One. 2008. 3(10): e3511.

[17]. Tufail Y, Matyushov A, Baldwin N, et al. Transcranial pulsed ultrasound stimulates intact brain circuits. Neuron. 2010. 66(5): 681-694.

[18]. Streit W J. Microglia as neuroprotective, immunocompetent cells of the CNS. Glia. 2002. 40(2): 133-139.

[19]. Alonso A, Reinz E, Fatar M, et al. Clearance of albumin following ultrasound-induced blood - brain barrier opening is mediated by glial but not neuronal cells. Brain Research. 2011. 1411:9-16.

[20]. M M T, Milla K, Maria P, et al. Bone-marrow-derived cells contribute to the recruitment of microglial cells in response to beta-amyloid deposition in APP/PS1 double transgenic Alzheimer mice. Neurobiology of Disease. 2005. 18(1):134-42.

[21]. R S A, Serge R. Role of inflammation in the neurobiology of stem cells. NeuroReport. 2005. 15(15): 2305-10.

[22]. Hong S, Beja-Glasser V F, Nfonoyim B M, et al. Complement and microglia mediate early synapse loss in Alzheimer mouse models. Science. 2016. 352(6286): 712-716.

[23]. Daria A, Colombo A, Llovera G, et al. Young microglia restore amyloid plaque clearance of aged microglia. EMBO J. 2016. 36(5):583-603.

[24]. Simard A R, Soulet D, Gowing G, et al. Bone Marrow-Derived Microglia Play a Critical Role in Restricting Senile Plaque Formation in Alzheimer's Disease. Neuron. 2006. 49(4): 489-502.

[25]. Jordão J F, Thévenot E, Markham-Coultes K, et al. Amyloid- $\beta$ plaque reduction, endogenous antibody delivery and glial activation by brain-targeted, transcranial focused ultrasound. Experimental Neurology. 2013. 248: 16-29.

[26]. Bocchi L, Branca J J V, Pacini S, et al. Effect of ultrasounds on neurons and microglia: Cell viability and automatic analysis of cell morphology. Biomedical Signal Processing and Control. 2015. 22: 44-53. 